\title{
Comparison of Exenatide and Metformin Monotherapy in Overweight/Obese Patients with Newly Diagnosed Type 2 Diabetes
}

\author{
Jia Liu, Yanjin Hu, Yuan Xu, Yumei Jia, Li Miao, and Guang Wang \\ Department of Endocrinology, Beijing Chao-Yang Hospital, Capital Medical University, No. 8, Gongti South Road, \\ Chaoyang District, Beijing 100020, China \\ Correspondence should be addressed to Guang Wang; drwg6688@126.com
}

Received 7 May 2017; Revised 19 August 2017; Accepted 7 September 2017; Published 20 November 2017

Academic Editor: Mario Maggi

Copyright (C) 2017 Jia Liu et al. This is an open access article distributed under the Creative Commons Attribution License, which permits unrestricted use, distribution, and reproduction in any medium, provided the original work is properly cited.

\begin{abstract}
Aims. The present study assessed the therapeutic effect of exenatide and metformin as the initial therapy on overweight/obese patients with newly diagnosed type 2 diabetes (T2D). Methods. The prospective, nonrandomized, interventional study enrolled a total of 230 overweight or obese patients with newly diagnosed T2D who were administrated exenatide or metformin hydrochloride for 12 weeks. Results. 224/230 patients, including 106 in the exenatide group and 118 in the metformin group, completed the 12-week treatment. Both exenatide and metformin significantly decreased the HbA1c levels in overweight/obese patients with newly diagnosed T2D (all $P<0.05$ ). The reduction in HbA1c and the proportion of patients with $\mathrm{HbA1c}<7.0 \%$ $(53 \mathrm{mmol} / \mathrm{mol})$ were higher in the exenatide group than in the metformin group (all $P<0.05)$. The exenatide treatment caused a greater decline in the body weight and BMI as compared to the metformin treatment (all $P<0.01$ ). The exenatide treatment $(\beta=0.41, P<0.01)$ and baseline HbA1c level $(\beta=-0.84, P<0.01)$ were independent influencing factors for the decrease in HbA1c level. Conclusions. For an initial therapy in overweight/obese patients with newly diagnosed T2D, exenatide causes a better glycemic control than metformin. This trial is registered with NCT03297879.
\end{abstract}

\section{Introduction}

Type 2 diabetes (T2D) is a common metabolic disease with high morbidity and mortality due to T2D-related complications [1]. Obesity is a major risk factor for T2D as it induces chronic inflammation, endoplasmic reticulum stress, mitochondrial dysfunction, and insulin resistance [2]. The impact on body weight is also considered a critical aspect of the clinical evaluation of hypoglycemic drugs. Several studies have confirmed that some hypoglycemic drugs like sulfonylureas, thiazolidinediones, or insulin tend to cause gain weight, while metformin leads to no change or mild decline in body weight [3]. Thus, metformin is frequently used as a firstchoice therapy in overweight/obese T2D patients [3]. Glucagon-like peptide-1 (GLP-1) receptor agonist is a novel agent approved for treating T2D and has been demonstrated to induce significant weight loss in overweight/obese T2D patients [3-6].
However, it is yet unclear whether as initial treatment, GLP-1 receptor agonist contributes to an improved therapeutic efficacy than metformin in overweight/obese patients with newly diagnosed T2D. Exenatide is a classical drug of a GLP-1 receptor agonist. In the present study, we assessed the therapeutic effect of exenatide and metformin as the initial therapy on overweight/obese patients with newly diagnosed T2D.

\section{Materials and Methods}

2.1. Subjects. The present study was a prospective, nonrandomized, interventional study, performed between September 2013 and October 2015 at the Department of Endocrinology in Beijing Chao-Yang Hospital affiliated to Capital Medical University. We consecutively enrolled 230 drug-naïve, overweight, or obese patients with newly diagnosed T2D, who fulfilled the following inclusion criteria: (1) age 20 to 
$<65$ years, (2) body mass index (BMI) $\geq 24 \mathrm{~kg} / \mathrm{m}^{2}$ [7], and (3) $\mathrm{HbAlc} \geq 7 \%(53 \mathrm{mmol} / \mathrm{mol})$. All of the patients have no diabetes antibodies and were diagnosed with T2D within the previous 3 months, according to the ADA diagnostic criteria [3]. None of the patients had been administered antidiabetic drugs or diet therapy before participation. Neither of the patients presented any history of pancreatitis, coronary artery disease, liver function impairment, renal function impairment, intestinal surgery, chronic hypoxic diseases (emphysema and cor pulmonale), infectious disease, hematological disease, systemic inflammatory disease, or cancer. Patients who were pregnant, possibly pregnant, or ingesting agents known to influence glucose or lipid metabolism were also excluded.

This study was conducted in accordance with the Declaration of Helsinki and approved by the Ethics Committee of Beijing Chao-Yang Hospital affiliated with Capital Medical University. All participants provided written informed consent.

2.2. Study Design. All participants were overweight or obese T2D patients, conforming to the indication of metformin or exenatide [3]. The patients were assigned to the exenatide group (110 patients) or metformin group (120 patients), respectively, according to their will. Exenatide was administrated with $5 \mu \mathrm{g}$ bid for 4 weeks and $10 \mu \mathrm{g}$ bid for 8 weeks. Metformin hydrochloride was initiated at a dose of $500 \mathrm{mg}$ bid for 2 weeks and added to $2.0 \mathrm{~g} /$ day for 8 weeks. All patients were provided lifestyle counseling upon enrollment.

Patients were followed up every 4 weeks. At each visit, anthropomorphic parameters and adverse events were recorded. Height and weight were measured to the nearest $0.1 \mathrm{~cm}$ and $0.1 \mathrm{~kg}$, respectively, by the same trained individuals. Minor hypoglycemia was defined as signs or symptoms confirmed by blood glucose $<3.9 \mathrm{mmol} / \mathrm{L}$ with a recovery after self-treatment. Major hypoglycemia was defined as signs or symptoms recovered after administration of carbohydrate, glucagon, or other resuscitative treatment or confirmed by blood glucose $<3.0 \mathrm{mmol} / \mathrm{L}$ with the assistance of another individual.

Laboratory assays were performed at baseline. After 12 weeks of exenatide or metformin treatment, blood samples were obtained in the morning after overnight fasting and stored at $-80^{\circ} \mathrm{C}$. Total cholesterol (TC), low-density lipoprotein cholesterol (LDL-C), high-density lipoprotein cholesterol (HDL-C), and triglycerides (TG) were measured by colorimetric enzymatic assays, using an autoanalyzer (Hitachi 7170). FBG, fasting insulin (FINS), and HbAlc were measured at the Central Chemistry Laboratory of Beijing Chao-Yang Hospital affiliated with Capital Medical University. BMI was calculated as weight $(\mathrm{kg}) /$ height $\left(\mathrm{m}^{2}\right)$. HOMA of insulin resistance (HOMA-IR) and homeostasis model assessment of $\beta$-cell function (HOMA- $\beta$ ) have been computed according to the following formulas: HOMA$\mathrm{IR}=\mathrm{FINS}(\mu \mathrm{IU} / \mathrm{mL}) \times \mathrm{FBG}(\mathrm{mmol} / \mathrm{L}) / 22.5$ and HOMA$\beta=20 \times$ FINS $(\mu \mathrm{IU} / \mathrm{mL}) / \mathrm{FBG}(\mathrm{mmol} / \mathrm{L})-3.5[8]$.

2.3. Statistical Analysis. Data were analyzed using SPSS 17.0 (SPSS Inc., Chicago, IL, USA). Continuous data were expressed as mean \pm standard deviation (SD). In the event of failure to follow a normal distribution, the values of TG, FINS, HOMA-IR, and HOMA- $\beta$ were presented as medians (the upper and lower quartiles). Variables that were nonnormally distributed were log-transformed before analysis. Comparisons between the groups were assessed by the independent sample $t$-test and ANOVA test. The differences of proportions were analyzed by the chi-square test. The changes in parameters from baseline within groups were evaluated using the two-tailed paired $t$-test. Multivariate regression analysis was applied to assess the correlations between the change in $\mathrm{HbAlc}$ level and relevant variables. Statistical significance was inferred when $P<0.05$.

\section{Results}

3.1. Baseline Characteristics in the Exenatide and Metformin Groups. Table 1 presents the baseline characteristics of the exenatide and metformin groups. Age, gender, the proportion of obese patients, body weight, BMI, TC, LDL-C, HDL-C, TG, FBG, FINS, HbAlc, HOMA-IR, and HOMA$\beta$ were comparable in the two groups (all $P>0.05$ ).

3.2. Changes in Anthropometric Measurements after Exenatide or Metformin Treatment. During therapy, 4 patients (3.6\%) in the exenatide group dropped out of the study because of moderate-to-severe nausea and vomiting, whereas 2 patients (1.7\%) in the metformin group dropped out because of moderate-to-severe vomiting and diarrhea.

After the 12-week treatment, significant reductions in body weight and BMI were observed in the exenatide and metformin groups (all $P<0.05$ ), respectively (Table 2). However, the exenatide treatment demonstrated a greater declination in the body weight and BMI as compared to the metformin treatment (all $P<0.01$ ) (Table 2).

3.3. Alterations in Glucose Metabolic Parameters after Exenatide or Metformin Treatment. Both exenatide and metformin treatments significantly decreased the FBG and HbAlc levels at 12 weeks (all $P<0.05$ ) (Table 2); however, no difference was observed in the reductions in FBG between exenatide and metformin treatments. Nevertheless, the $\mathrm{HbA1c}$ levels in the exenatide group were reduced more than those in the metformin group [ $-2.81(-3.42$ to 2.45$)$ versus -1.44 ( -1.67 to 1.21$), P<0.01$ ] (Table 2). The proportion of patients with $\mathrm{HbAlc}<7.0 \%(53 \mathrm{mmol} / \mathrm{mol})$ in the exenatide group was $79.5 \%$, which was higher than the $64.0 \%$ in the metformin group $(P<0.05)$ (Table 2) [3].

We also observed a significant increase in HOMA- $\beta$ in the two groups after exenatide or metformin treatment (all $P<0.05$ ) (Table 2). The increase in the HOMA- $\beta$ value of the exenatide group was higher than that of the metformin group [33.01 (20.19-45.82) versus 14.03 (6.34-21.72), $P<0.01]$. However, the decrease in the HOMA-IR value was similar after 12 weeks of exenatide or metformin treatment $(P>0.05)$ (Table 2).

Interestingly, exenatide did not cause a greater decrease in the HbAlc level than metformin after adjustment for the reduced body weight following the 12 -week treatment 
TABLE 1: Baseline characteristics of the exenatide and metformin groups.

\begin{tabular}{lccc}
\hline Parameters & Exenatide $(n=110)$ & Metformin $(n=120)$ & $P$ \\
\hline Age, $y$ & $45.77 \pm 10.60$ & $48.05 \pm 8.04$ & 0.097 \\
Gender, males/females, $n$ & $64 / 46$ & $71 / 49$ & 0.880 \\
Overweight/obesity, $n$ & $57 / 53$ & $77.98 \pm 11.88$ & 0.918 \\
Body weight, kg & $78.76 \pm 10.01$ & $27.57 \pm 3.56$ & 0.907 \\
BMI, kg/m & $27.83 \pm 2.38$ & $5.29 \pm 1.22$ & 0.815 \\
TC, mmol/L & $5.21 \pm 1.25$ & $3.05 \pm 0.90$ & 0.664 \\
LDL-C, mmol/L & $3.07 \pm 0.89$ & $1.24 \pm 0.29$ & 0.864 \\
HDL-C, mmol/L & $1.12 \pm 0.43$ & $1.93(1.32-2.75)$ & 0.087 \\
TG, mmol/L & $2.07(1.27-3.99)$ & $9.10 \pm 1.34$ & 0.235 \\
FBG, mmol/L & $9.34 \pm 3.27$ & $10.14(5.25-14.94)$ & 0.594 \\
FINS, $\mu$ IU/mL & $9.50(5.98-14.37)$ & $9.03 \pm 1.18$ & 0.337 \\
HbAlc, $\%$ & $9.11 \pm 1.46$ & $3.87(2.27-6.02)$ & 0.230 \\
HOMA-IR & $3.94(2.47-5.93)$ & $50.55(29.80-74.81)$ & 0.638 \\
HOMA- $\beta$ & $55.13(29.64-76.65)$ & 0.289 \\
\hline
\end{tabular}

Data are means \pm SD unless indicated otherwise. TG, FINS, HOMA-IR, and HOMA- $\beta$ are shown as medians (upper and lower quartiles). BMI: body mass index; TC: total cholesterol; LDL-C: low-density lipoprotein cholesterol; HDL-C: high-density lipoprotein cholesterol; TG: triglyceride; FBG: fasting blood glucose; FINS: fasting insulin; HOMA-IR: homeostasis model assessment of insulin resistance; HOMA- $\beta$ : homeostasis model assessment of $\beta$-cell function.

TABLE 2: Changes in metabolic parameters after metformin or exenatide treatment.

\begin{tabular}{lccc}
\hline Parameters & Exenatide $(n=106)$ & Metformin $(n=118)$ & $P$ \\
\hline Body weight, kg & $-5.79(-8.09$ to -3.48$)$ & $-2.30(-0.99$ to -0.66$)$ & 0.001 \\
BMI, $\mathrm{kg} / \mathrm{m}^{2}$ & $-1.99(-2.85$ to -1.14$)$ & $-0.83(-0.99$ to -0.66$)$ & $-0.35(-0.52$ to -0.19$)$ \\
TC, $\mathrm{mmol} / \mathrm{L}$ & $-0.58(-1.00$ to -0.15$)$ & $-0.14(-0.26$ to -0.03$)$ & 0.252 \\
LDL-C, $\mathrm{mmol} / \mathrm{L}$ & $-0.17(-0.42$ to 0.08$)$ & $-0.04(-0.08$ to 0.00$)$ & 0.900 \\
HDL-C, $\mathrm{mmol} / \mathrm{L}$ & $0.06(-0.02$ to 0.13$)$ & $-0.03(-0.31$ to 0.26$)$ & 0.358 \\
TG, $\mathrm{mmol} / \mathrm{L}$ & $-1.26(-2.07$ to -0.45$)$ & $-2.17(-2.51$ to -1.73$)$ & 0.000 \\
FBG, $\mathrm{mmol} / \mathrm{L}$ & $-2.62(-3.89$ to -1.35$)$ & $-2.03(-4.50$ to -1.52$)$ & 0.321 \\
FINS, $\mu \mathrm{IU} / \mathrm{mL}$ & $0.63(-1.19$ to 2.46$)$ & $-1.44(-1.67$ to -1.21$)$ & 0.000 \\
HbAlc, $\%$ & $-2.81(-3.42$ to -2.45$)$ & $14.03(6.34-21.72)$ & 0.000 \\
HOMA-IR & $-2.05(-2.69$ to -1.37$)$ & $75(64.0)$ & 0.565 \\
HOMA- $\beta$ & $33.01(20.19-45.82)$ & $84(79.5)$ & 0.002 \\
HbA1C $<7.0 \%, n(\%)$ & & 0.033 \\
\hline
\end{tabular}

Data are shown as difference $(95 \% \mathrm{CI})$ versus baseline, except for $\mathrm{HbA1C}<7.0 \%$. BMI: body mass index; TC: total cholesterol; LDL-C: low-density lipoprotein cholesterol; HDL-C: high-density lipoprotein cholesterol; TG: triglyceride; FBG: fasting blood glucose; FINS: fasting insulin; HOMA-IR: homeostasis model assessment of insulin resistance; HOMA- $\beta$ : homeostasis model assessment of $\beta$-cell function; $\mathrm{HbA1C}<7.0 \%$ : the proportion of patients with $\mathrm{HbA1c}<7.0 \%$ (53 $\mathrm{mmol} / \mathrm{mol})$.

$(P>0.05)$. However, the greater increase in the HOMA- $\beta$ value was still observed in the exenatide group than in the metformin group postadjustment for the decrease in FBG $(P<0.05)$.

\subsection{Changes in Lipid Profile after Exenatide or Metformin} Treatment. Both exenatide and metformin significantly reduced the TC levels after 12 weeks of treatment $(P<0.05)$ (Table 2). Moreover, a significant decrease in the plasma TG levels of the exenatide group and LDL-C levels of the metformin group was also observed posttreatment (all $P<$ 0.05 ) (Table 2). In the exenatide group, the TG levels showed a greater decline as compared to those in the metformin treatment group $(P<0.01)$ (Table 2$)$.
3.5. Multivariate Regression Analysis to Find the Correlation between the Decrease in HbAlc Level and Relevant Variables. Multivariate regression analysis was used to further assess the relationships between the decrease in the HbAlc level and related variables. The influence of six variables such as age, gender, body mass index, baseline HbAlc, baseline FBG, and drug treatment was assessed. The analysis indicated that the exenatide treatment $(\beta=0.41, P<0.01)$ and baseline HbA1c level $(\beta=-0.84, P<0.01)$ were independent influencing factors for the decrease in HbA1c level.

3.6. Adverse Events. During the 12-week treatment with exenatide or metformin, no major hypoglycemia was observed in any patient (Table 3). Incidences of minor hypoglycemia 
TABLE 3: Adverse events in the exenatide and metformin groups.

\begin{tabular}{lccc}
\hline Adverse events & $\begin{array}{c}\text { Exenatide } \\
(n=106)\end{array}$ & $\begin{array}{c}\text { Metformin } \\
(n=118)\end{array}$ & $P$ \\
\hline $\begin{array}{l}\text { Major hypoglycemia, } \\
\text { (\%) }\end{array}$ & 0 & 0 & 1 \\
$\begin{array}{l}\text { Minor hypoglycemia, } \\
n(\%)\end{array}$ & $5(4.7 \%)$ & $3(2.5 \%)$ & 0.481 \\
\hline
\end{tabular}

occurred in 5/106 (4.7\%) and 3/118 (2.5\%) patients treated with exenatide and metformin, respectively (Table 3 ).

\section{Discussion}

In the present study, both exenatide and metformin significantly decreased the HbA1c levels in overweight/obese patients with newly diagnosed T2D. After the 12-week treatment, a reduction in $\mathrm{HbAlc}$ and the proportion of patients with $\mathrm{HbA} 1 \mathrm{c}<7.0 \%(53 \mathrm{mmol} / \mathrm{mol})$ were higher in the exenatide group than in the metformin group. The exenatide treatment caused a greater decline in the body weight and BMI as compared to the metformin treatment. Multivariate regression analysis indicated that the exenatide treatment and baseline HbAlc level were independent influencing factors for the decrease in HbAlc level.

In our study, metformin reduced the FBG levels by $2.17 \mathrm{mmol} / \mathrm{L}$ and the HbA1c levels by $1.44 \%$ from baseline, which was consistent with previous studies $[9,10]$. However, in the exenatide group, the mean changes in FBG and HbA1c from baseline were $-2.62 \mathrm{mmol} / \mathrm{L}$ and $-2.81 \%$, respectively, higher than the decrease in other studies $[4-6,10]$. One possible explanation is that our study assessed the initial therapy of exenatide, and the previous studies mainly evaluated exenatide as the add-on treatment in patients with suboptimum glycemic control after the initial therapy [4-6, 10]. As the preliminary treatment, the improvement of metabolic disorders is associated with the effect of exenatide and lifestyle modification [11]. Moreover, overweight/obese patients with T2D might present a better lifestyle modification if treated with exenatide, which can cause the slowing of gastric emptying and suppression of appetite [12]. Another reason might be associated with the differences in the race [13]. Western population with $\mathrm{T} 2 \mathrm{D}$ showed a significant reduction in the GLP-1 sensitivity; however, no significant reduction was seen in Asians with T2D $[14,15]$. Thus, the preserved GLP-1 sensitivity might account for the greater glucose-lowering efficacy of exenatide treatment in our study $[14,15]$. In addition, Chinese T2D patients have higher postprandial glucose levels than Caucasians, and exenatide has a greater effect on postprandial glucose by stimulating glucose-dependent insulin secretion, inhibiting postprandial glucagon, and delaying the gastric discharging $[12,16]$. In our study, exenatide treatment resulted in a greater reduction in HbAlc levels than metformin treatment. The proportion of patients with $\mathrm{HbA} 1 \mathrm{c}<7.0 \%(53 \mathrm{mmol} / \mathrm{mol})$ in the exenatide group was $79.5 \%$, which was higher than the $64.0 \%$ in the metformin group. Multivariate regression analysis indicated that the exenatide treatment was an independent influencing factor for the decrease in HbAlc level. These results are consistent with those of a meta-analysis, which demonstrated that GLP-1 receptor agonists contribute to a larger proportion of patients achieving $\mathrm{HbAlc}<7.0 \%$ (53 $\mathrm{mmol} / \mathrm{mol})$ than metformin [17]. Thus, the results might suggest that for initial therapy in overweight/obese patients with newly diagnosed T2D, exenatide causes a better glycemic control than metformin.

Obesity is associated with T2D by inducing endoplasmic reticulum stress, chronic inflammatory state, and insulin resistance [2]. Our study demonstrated that exenatide and metformin individually caused a significant decrease in body weight. A greater weight loss was observed in the exenatide group compared to the metformin group. These results for metformin are consistent with those of our previous and other studies $[9,18]$. Metformin leads to a moderate weight loss by several mechanisms, including increasing insulin sensitivity, decreasing gastrointestinal absorption of carbohydrates, and reducing ghrelin and leptin levels after glucose overload [19]. In addition, metformin increased circulating levels of active forms of GLP-1, which might be involved in the suppression of appetite [20]. In the present study, exenatide significantly decreased the body weight by $5.79 \mathrm{~kg}$. Exenatide lowers body weight by slowing gastric emptying, promoting satiety, and decreasing food intake [12]. In addition, lifestyle intervention, especially diet control, can also effectively reduce the body weight in patients with T2D [21]. Due to the suppression of appetite, GLP-1 receptor agonist might exhibit a synergistic effect with life intervention and contribute to improved diet control and hence more weight loss. Notably, after adjustment for the decrease in body weight, no significant difference was observed in the reduction in HbAlc between the exenatide and metformin treatments. This suggested that the better hypoglycemic effect of exenatide on overweight/obese patients might be primarily associated with enhanced weight loss.

Insulin resistance plays a major role in the pathogenesis of T2D and its complications [2]. Metformin has been widely established as a strong insulin sensitizer [3]. The present study showed that exenatide and metformin similarly decreased insulin resistance in overweight/obese patients with T2D. The significant reduction in body weight is one of the key reasons for the improvement of insulin resistance of exenatide and metformin [22]. Both GLP-1 receptor agonist and metformin exerted some beneficial effects on insulin resistance indirectly by inhibiting oxidative stress and regulating lipid metabolism $[23,24]$. Thus, it might suggest that exenatide is another insulin sensitizer that leads to amelioration of insulin resistance in obese patients with T2D.

Compared to the Western population, Asians with T2D showed a distinct $\beta$-cell dysfunction [25]. In the present study, both exenatide and metformin treatments significantly increased HOMA- $\beta$ values, and the increase in the exenatide group was higher than that in the metformin group. The alleviation of glucose toxicity, rather than a direct drug effect, might underlie the HOMA- $\beta$ improvement in the two groups [26]. However, after adjustment for the decrease in FBG, the increase in the HOMA- $\beta$ value was still observed in the exenatide group than in the metformin group. 
Previous studies showed that metformin indirectly increased $\beta$-cell function through decreasing glucotoxicity, lowering insulin resistance, inhibiting the formation of advanced glycation end products, and reducing oxidative stress $[23,26]$. In addition to the similar capabilities of metformin, exenatide also displays some direct beneficial effects on islet $\beta$-cells [27-30]. A previous study showed that exenatide improved the insulin secretion of $\beta$-cells as compared to the insulin glargine in a similar glycemic control, and this effect was sustained after a 4-week off-drug period [28]. Animal studies have demonstrated that GLP-1 promotes the proliferation of $\beta$-cells and inhibits their apoptosis $[29,30]$. Cell-based studies showed that GLP-1 enhanced the viability and inhibited the mitochondrial-dependent apoptosis in the PKCdependent pathway [30].

Notably, our study has several limitations. The major limitation was that the present study was not a randomized controlled trial. In addition, our study also lacked accurate methods, such as hyperglycemic and hyperinsulinemic clamp techniques, to assess $\beta$-cell function and insulin sensitivity. Furthermore, the treatment duration was relatively short. Long-term observation of these treatments is needed to evaluate the persistence of beneficial effects over time. Moreover, the cost/benefit ratio is another aspect that should be considered. In terms of medical expenses, metformin might be more preferable for a lifelong treatment. Despite these limitations, one implication of the present study is that for a patient with newly diagnosed T2D, exenatide might contribute to a better effect on glycemic control, weight management, and $\beta$-cell function, which at least partly represents an avenue for investigation in the treatment of T2D.

In conclusion, for an initial therapy in overweight/obese patients with newly diagnosed T2D, exenatide causes a better glycemic control than metformin, whose mechanism might potentially be related to the better weight loss effect and amelioration of $\beta$-cell function.

\section{Conflicts of Interest}

No competing financial interests exist.

\section{Acknowledgments}

This work was supported by grants from the Capital Clinical Research Foundation of Beijing Municipal Commission of Science and Technology (no. Z161100000516069) to Guang Wang, and National Natural Science Foundation of China (no. 81600657), and Beijing Municipal Administration of Hospitals' Youth Programme (no. QML20150308) to Jia Liu.

\section{References}

[1] L. Guariguata, D. R. Whiting, I. Hambleton, J. Beagley, U. Linnenkamp, and J. E. Shaw, "Global estimates of diabetes prevalence for 2013 and projections for 2035," Diabetes Research and Clinical Practice, vol. 103, no. 2, pp. 137-149, 2014.

[2] A. J. Garber, "Obesity and type 2 diabetes: which patients are at risk?," Diabetes, Obesity and Metabolism, vol. 14, no. 5, pp. 399-408, 2012.
[3] American Diabetes Association, "Standards of medical care in diabetes-2013," Diabetes Care, vol. 36, Supplement 1, pp. S11-S66, 2013.

[4] G. Derosa, I. G. Franzetti, F. Querci et al., "Exenatide plus metformin compared with metformin alone on $\beta$-cell function in patients with type 2 diabetes," Diabetic Medicine, vol. 29, no. 12, pp. 1515-1523, 2012.

[5] M. Diamant, L. Van Gaal, B. Guerci et al., "Exenatide once weekly versus insulin glargine for type 2 diabetes (DURATION-3): 3-year results of an open-label randomised trial," The Lancet Diabetes and Endocrinology, vol. 2, no. 6, pp. 464-473, 2014.

[6] J. B. Buse, J. Rosenstock, G. Sesti et al., "Liraglutide once a day versus exenatide twice a day for type 2 diabetes: a 26-week randomised, parallel-group, multinational, open-label trial (LEAD-6)," The Lancet, vol. 374, no. 9683, pp. 39-47, 2009.

[7] Chinese Society of Endocrinology, "Chinese expert consensus for the prevention and treatment of obesity in adults," vol. 27, pp. 711-717, 2011.

[8] V. Bermudez, R. Cano, C. Cano et al., "Homeostasis model assessment (HOMA) as surrogate insulinization criteria in patients with type 2 diabetes," American Journal of Therapeutics, vol. 15, no. 4, pp. 409-416, 2008.

[9] G. Wang, J. Liu, N. Yang et al., "MARCH2: comparative assessment of therapeutic effects of acarbose and metformin in newly diagnosed type 2 diabetes patients," PLoS One, vol. 9, no. 8, article e105698, 2014.

[10] D. Russell-Jones, R. M. Cuddihy, M. Hanefeld et al., "Efficacy and safety of exenatide once weekly versus metformin, pioglitazone, and sitagliptin used as monotherapy in drug-naive patients with type 2 diabetes (DURATION-4): a 26-week double-blind study," Diabetes Care, vol. 35 , no. 2, pp. 252258, 2012.

[11] A. P. Cotter, N. Durant, A. A. Agne, and A. L. Cherrington, "Internet interventions to support lifestyle modification for diabetes management: a systematic review of the evidence," Journal of Diabetes and its Complications, vol. 28, no. 2, pp. 243-251, 2014.

[12] D. J. Drucker and M. A. Nauck, "The incretin system: glucagon-like peptide-1 receptor agonists and dipeptidyl peptidase-4 inhibitors in type 2 diabetes," The Lancet, vol. 368, no. 9548, pp. 1696-1705, 2006.

[13] Y. G. Kim, S. Hahn, T. J. Oh, K. S. Park, and Y. M. Cho, "Differences in the HbAlc-lowering efficacy of glucagon-like peptide-1 analogues between Asians and non-Asians: a systematic review and meta-analysis," Diabetes, Obesity \& Metabolism, vol. 16, no. 10, pp. 900-909, 2014.

[14] Y. M. Cho, "Incretin physiology and pathophysiology from an Asian perspective," Journal of Diabetes Investigation, vol. 6, no. 5, pp. 495-507, 2015.

[15] T. J. Oh, M. Y. Kim, J. Y. Shin et al., "The incretin effect in Korean subjects with normal glucose tolerance or type 2 diabetes," Clinical Endocrinology, vol. 80, no. 2, pp. 221-227, 2014.

[16] W. H. Herman, K. M. Dungan, B. H. R. Wolffenbuttel et al., "Racial and ethnic differences in mean plasma glucose, hemoglobin A1c, and 1,5-anhydroglucitol in over 2000 patients with type 2 diabetes," The Journal of Clinical Endocrinology \&Metabolism, vol. 94, no. 5, pp. 1689-1694, 2009.

[17] K. Esposito, P. Chiodini, G. Bellastella, M. I. Maiorino, and D. Giugliano, "Proportion of patients at HbA1c target $<7 \%$ with eight classes of antidiabetic drugs in type 2 diabetes: 
systematic review of 218 randomized controlled trials with 78 945 patients," Diabetes, Obesity \& Metabolism, vol. 14, no. 3, pp. 228-233, 2012.

[18] W. C. Knowler, E. Barrett-Connor, S. E. Fowler et al., "Reduction in the incidence of type 2 diabetes with lifestyle intervention or metformin," The New England Journal of Medicine, vol. 346, no. 6, pp. 393-403, 2002.

[19] S. K. Thondam, A. Cross, D. J. Cuthbertson, J. P. Wilding, and C. Daousi, "Effects of chronic treatment with metformin on dipeptidyl peptidase- 4 activity, glucagon-like peptide 1 and ghrelin in obese patients with type 2 diabetes mellitus," Diabetic Medicine, vol. 29, no. 8, pp. e205-e210, 2012.

[20] E. Mannucci, A. Ognibene, F. Cremasco et al., "Effect of metformin on glucagon-like peptide 1 (GLP-1) and leptin levels in obese nondiabetic subjects," Diabetes Care, vol. 24, no. 3, pp. 489-494, 2001.

[21] E. P. de Oliveira, A. C. Diegoli, J. E. Corrente, K. C. McLellan, and R. C. Burini, "The increase of dairy intake is the main dietary factor associated with reduction of body weight in overweight adults after lifestyle change program," Nutrición Hospitalaria, vol. 32, no. 3, pp. 1042-1049, 2015.

[22] M. Sene-Fiorese, F. O. Duarte, A. E. de Aquino Junior et al., "The potential of phototherapy to reduce body fat, insulin resistance and "metabolic inflexibility" related to obesity in women undergoing weight loss treatment," Lasers in Surgery and Medicine, vol. 47, no. 8, pp. 634-642, 2015.

[23] B. Batchuluun, T. Inoguchi, N. Sonoda et al., "Metformin and liraglutide ameliorate high glucose-induced oxidative stress via inhibition of PKC-NAD(P)H oxidase pathway in human aortic endothelial cells," Atherosclerosis, vol. 232, no. 1, pp. 156-164, 2014.

[24] R. Barazzoni, M. Zanetti, G. Gortan Cappellari et al., "Fatty acids acutely enhance insulin-induced oxidative stress and cause insulin resistance by increasing mitochondrial reactive oxygen species (ROS) generation and nuclear factor- $\kappa \mathrm{B}$ inhibitor $(\mathrm{I} \kappa \mathrm{B})$-nuclear factor- $\kappa \mathrm{B}(\mathrm{NF} \kappa \mathrm{B})$ activation in rat muscle, in the absence of mitochondrial dysfunction," Diabetologia, vol. 55, no. 3, pp. 773-782, 2012.

[25] A. Morimoto, Y. Tatsumi, K. Deura et al., "Impact of impaired insulin secretion and insulin resistance on the incidence of type 2 diabetes mellitus in a Japanese population: the Saku study,” Diabetologia, vol. 56, no. 8, pp. 1671-1679, 2013.

[26] R. Retnakaran and B. Zinman, "Short-term intensified insulin treatment in type 2 diabetes: long-term effects on $\beta$-cell function," Diabetes, Obesity \& Metabolism, vol. 14, Supplement 3, pp. 161-166, 2012.

[27] M. Zander, S. Madsbad, J. L. Madsen, and J. J. Holst, "Effect of 6-week course of glucagon-like peptide 1 on glycaemic control, insulin sensitivity, and $\beta$-cell function in type 2 diabetes: a parallel-group study," The Lancet, vol. 359, no. 9309, pp. 824-830, 2002.

[28] M. C. Bunck, A. Corner, B. Eliasson et al., "Effects of exenatide on measures of $\beta$-cell function after 3 years in metformintreated patients with type 2 diabetes," Diabetes Care, vol. 34, no. 9, pp. 2041-2047, 2011.

[29] J. Buteau, M. L. Spatz, and D. Accili, "Transcription factor FoxO1 mediates glucagon-like peptide-1 effects on pancreatic $\beta$-cell mass," Diabetes, vol. 55, no. 5, pp. 1190-1196, 2006.

[30] L. Zhang, Y. Wang, J. Wang, Y. Liu, and Y. Yin, "Protein kinase $\mathrm{C}$ pathway mediates the protective effects of glucagonlike peptide- 1 on the apoptosis of islet $\beta$-cells," Molecular Medicine Reports, vol. 12, no. 5, pp. 7589-7594, 2015. 


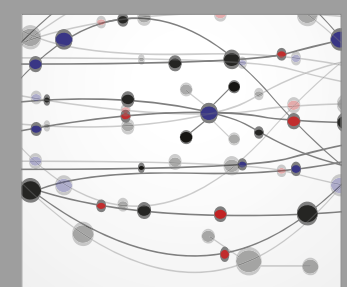

The Scientific World Journal
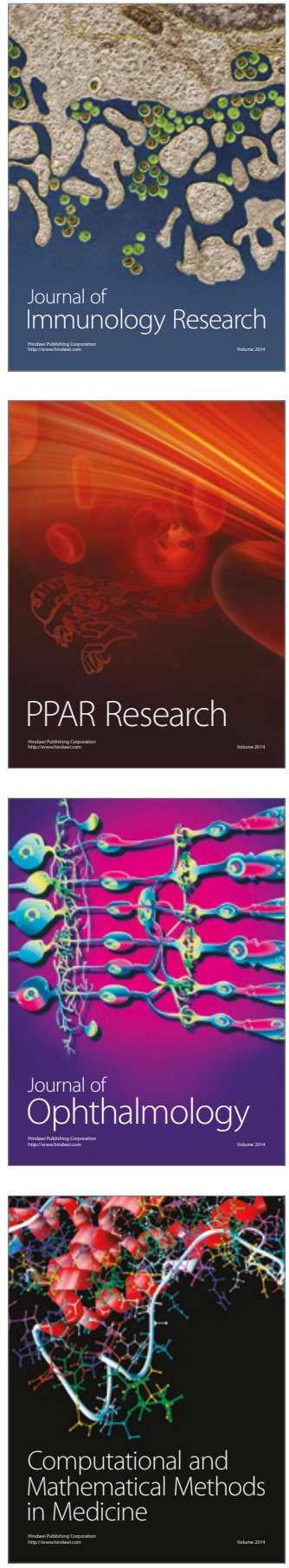

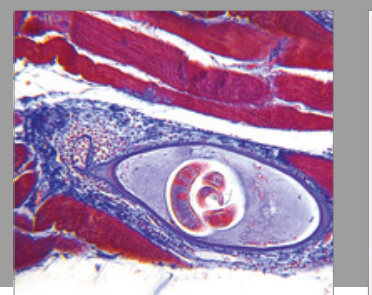

Gastroenterology Research and Practice
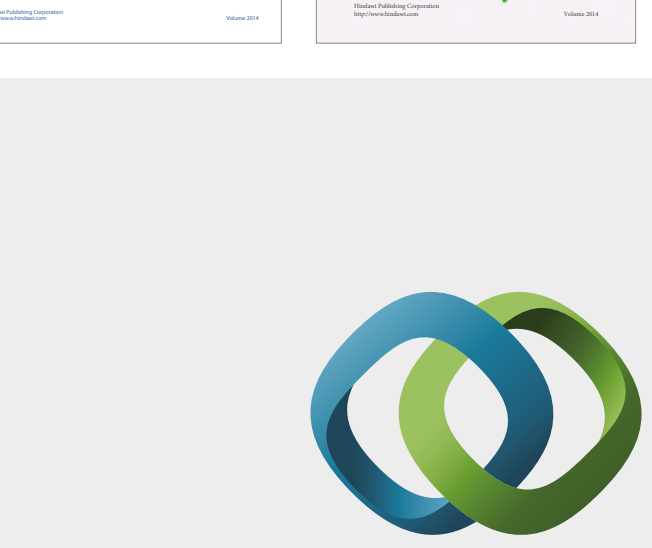

\section{Hindawi}

Submit your manuscripts at

https://www.hindawi.com
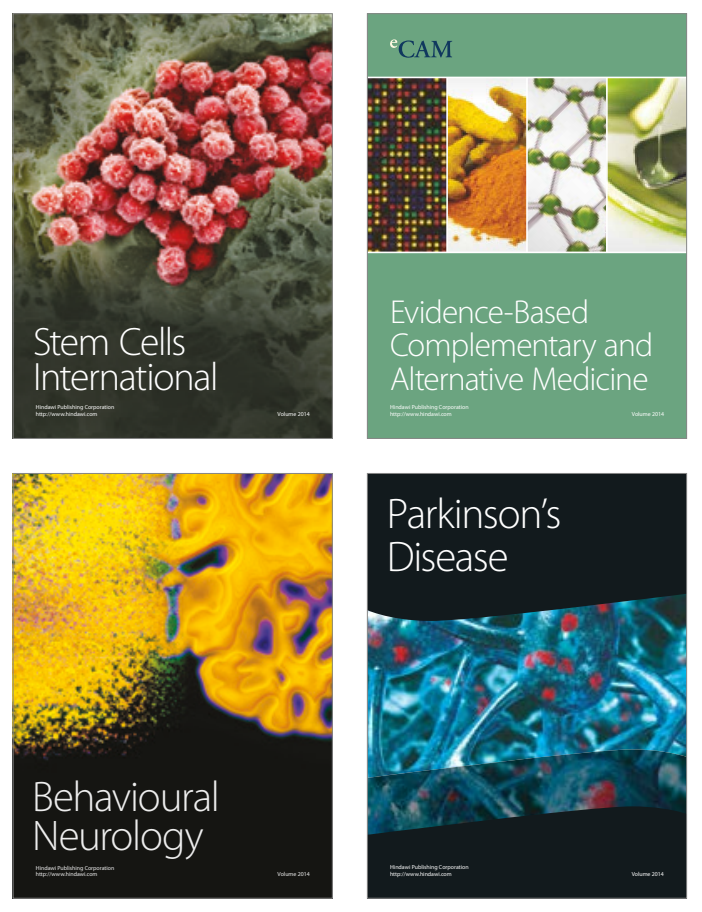
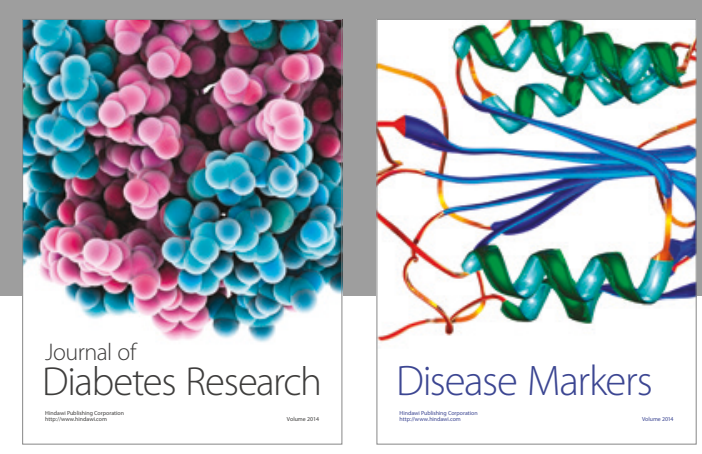

Disease Markers
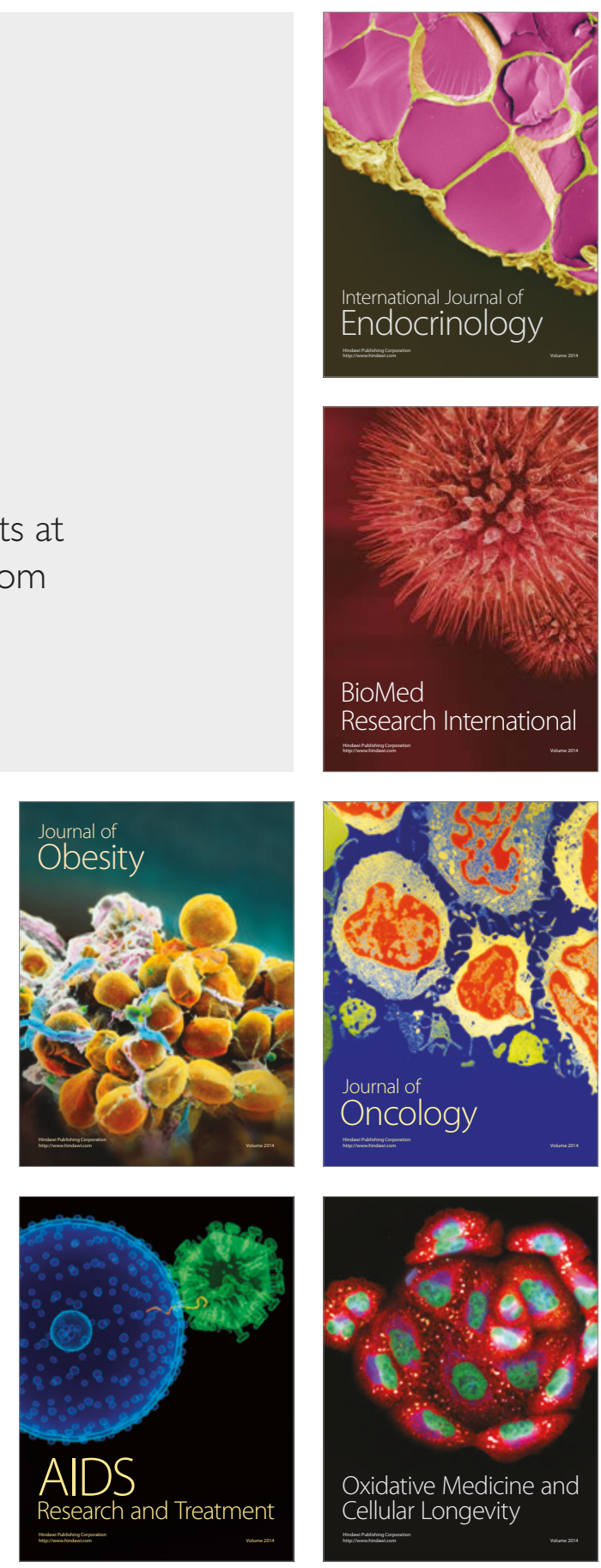